УДК 629.735.02:681.518.5

DOI: 10.15587/2313-8416.2014.33560

\title{
ДИАГНОСТИКА ТИПОВЫХ ОТКАЗОВ ПРИВОДЯЩИХ К ВОЗНИКНОВЕНИЮ ОСОБОЙ СИТУАЦИИ В ПОЛЕТЕ
}

\author{
(ㄱ) В. Н. Казак, Д. О. Шевчук, М. А. Васильев
}

В данной статье анализируются типовые отказы систем и элементов самолета приводящие к возникновению аварийной ситуации в полете. Приведены статистические данные о наиболее часто возникающих отказах управляющих поверхностей. Рассматривается три типа отказов: отказы датчиков, приводов и компонентов системы, диагностика их влияния на систему управления самолетом. Разработана методика диагностики отказов в полете

Ключевые слова: самолет, особая ситуация, отказы, система управления, реконфигурация, поверхности управления, повреждения

This article is about typical aircraft system and components faults that cause to extra situation in flight. It is given statistic data about common control surfaces faults. It is analyzed three main types of faults: sensors faults, actuators, components faults and diagnostic how these faults affect on aircraft control system. It is developed fault diagnostics methodic in flight

Keywords: aircraft, extra situation, faults, control system, reconfiguration, control surfaces, diagnostics

\section{1. Введение}

Сложность решения проблемы обеспечения безопасности полетов беспрерывно возрастает в связи с повышением интенсивности использования авиационной техники, которая кроме известных влияний ведет к значительному увеличению вероятности столкновения с механическими, биологическими и электрическими формированиями, а также расширением круга выполняемых ею функциональных задач. Сравнительный анализ статистических данных ICAO показал, что $35 \%$ случаев потери летательных аппаратов связанные с отказами и повреждениями систем автоматического управления, причем главным образом с отказами поводов и повреждениями внешних обводов, а также управляющих поверхностей. Также, необходимо отметить чрезвычайно высокую быстротечность развития аварийной ситуации, которая в свою очередь требует мгновенного вмешательства в ситуацию для принятия необходимых управляющих действий относительно предотвращения ее развития или перерастание в катастрофическую. Все это обусловливает рост роли бортовых средств автоматического выявления повреждений внешних обводов и управляющих поверхностей ЛА в полете, разработки перспективных методов и систем автоматической реконфигурации управляющих действий, а также интеллектуальных систем поддержки принятия решений экипажем в условиях возникновения аварийной ситуации в полете.

\section{2. Постановка проблемы}

Изучение основных причин и видов отказов. Разработка системы обнаружения и диагностики отказов.

\section{3. Литературный обзор}

В [1] рассмотрены различные математические модели отказов. С целью математического моделирования, отказы могут быть классифицированы как аддитивные (отказы компонентов системы) и мультипликативные (отказы приводов и датчиков) [2]. Отказы так же могут быть классифицированы по своим временным характеристикам как резкий (возникающий мгновенно), зарождающийся (параметры изменяются медленно) и прерывистый (периодически появляющийся и исчезающий) [3]. Главные предложения по проектированию и делению на различные виды систем диагностики отказов изложены в [4].

\section{4. Диагностика отказов управляющих} поверхностей в полете

Рассмотрим процентное соотношение частоты возникновения отказов различных поверхностей управления самолета, представленных на рис. 1.

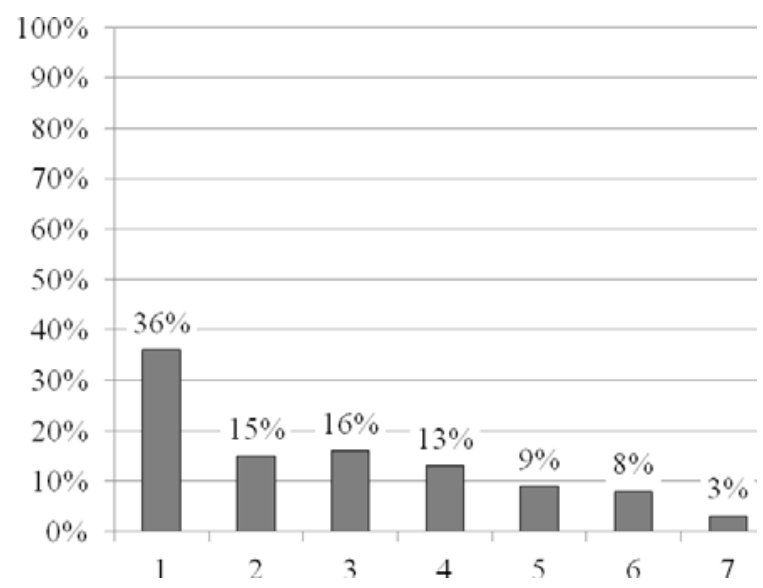

Рис. 1. Процентное отношение частоты отказа различных поверхностей управления: 1 - Закрылки; 2 - Руль направления; 3 - Руль высоты; 4 - Элероны;

5 - Спойлера; 6 - Стабилизаторы; 7 - Другое

Управление самолетом осуществляется при помощи управляющих поверхностей. При помощи двигателей управляют главным образом скоростью, в 
то время как управление по крену, тангажу и рысканию осуществляется при помощи элеронов, рулей направления и высоты.

Современные самолеты имеют больше поверхностей управления, а некоторые имеют специализированные поверхности управления. Разнообразие конфигурации и количества управляющих поверхностей обычно определяется требуемой производительностью, надежностью или спецификой предназначения. Так же для управления полетом используются канарды, подвижные законцовки крыла, флаппероны, спойлера и комбинации из основных управляющих поверхностей, таких как элевоны, флаппероны и стабилизаторы. Некоторые из них представлены на рис. 2.
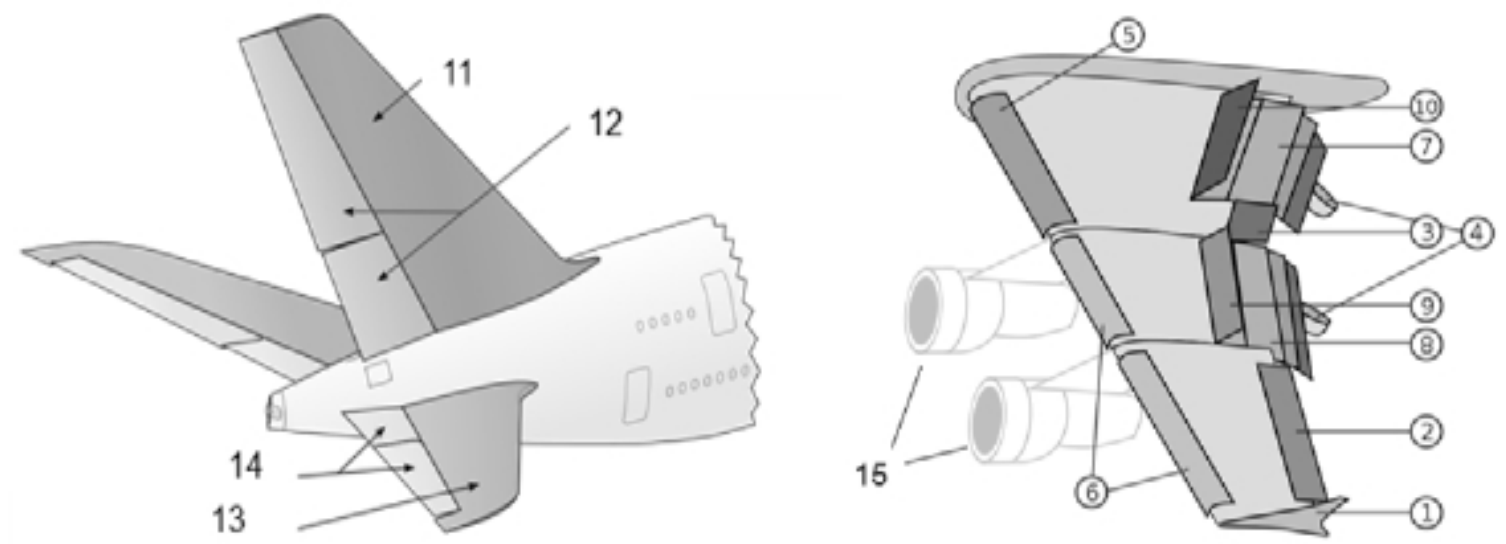

Рис. 2. Поверхности управления самолета: 1 - законцовка; 2 - элерон; 3 - высокоскоростной элерон; 4 - балки закрылок; 5 - предкрылок Крюгера; 6 - предкрылки; 7 - внутренние трёхщелевые закрылки; 8 - внешние трёхщелевые закрылки; 9 - интерцепторы (Спойлеры); 10 - элерон-интерцепторы; 11 - вертикальный стабилизатор; 12 - руль направления; 13 - горизонтальный стабилизатор; 14 - руль высоты; 15 - двигатели

Отклонение управляющих поверхностей осуществляется системой управления полетом и осуществляется при помощи электрического сервопривода или гидравлического сервопривода, или при помощи их комбинации.

Отклонение поверхностей управление создает изменение обтекающего потока воздуха, в результате чего происходит изменение аэродинамических сил и моментов. Эффект оказываемый поверхностями управления на вращательное и поступательное движение самолета может быть как желательным, так и рассматриваться как возмущение. К примеру, так как руль направления осуществляет управление по рысканью, то перекрестное управление вызывает нежелательный дополнительный крутящий момент. Некоторые из поверхностей управление могут быть использованы для создания дополнительного компенсирующего момента. Момент рысканья изменяется за счет парциального отклонения элеронов и спойлеров, которые создают разницу между двумя наборами. Управление по каналу крена так же осуществляется различными способами. Способность осуществлять управление по одному каналу различными управляющими поверхностями обуславливается избыточностью органов управления в системе. Эффективное применение этого свойства в разработке системы управления важнейшая задача современных систем отказоустойчивого управления.

Отказы - это нежелательные события, которые могут возникнуть в различных частях системы управления. В автоматической системе управления отказы классифицируются как отказы приводов, отказы сенсоров и отказы компонентов системы.
Отказы приводов бывают двух видов: частичная или полная потеря работоспособности. Полная потеря работоспособности привода может произойти в следствии поломки, короткого замыкания или разрыва обмотки, что в свою очередь может приводить к заклиниванию или не осуществлению действия согласно с управляющим сигналом. Один из наихудших случаев полного отказа приводов «заклинивание в крайнем положении», другой случай - «плавающий» отказ или потеря эффективности привода. Приводы частично потерявшие работоспособность выполняют лишь часть действий. Частичная потеря работоспособности может быть вызвана потерей пневматического или гидравлического давления возросшим сопротивлением или проседанием напряжения на источнике питания. Изза высокой стоимости и больших размеров и масс, дублирование приводов для повышения отказоустойчивости часто неприемлемо.

Отказы датчиков - это отказы, связанные с получением неверной информации с датчиков. Отказы датчиков также могут быть частичными (значения связанные с измеряемым параметром) и полные (значения которые не несут полезной информации). Из-за сравнительно малых размеров они могут быть продублированы для повышения отказоустойчивости.

Под отказами компонентов системы понимается отказы любых компонентов системы, так или иначе влияющих на управление или аэродинамические свойства самолета. Такие отказы обозначают изменение физических параметров самолета, таких как аэродинамические коэффициенты и вызваны чаще всего структурным 
повреждением. Отказы компонентов системы наиболее сложные для прогнозирования и парирования, так как включают в себя широкий класс непредвиденных ситуаций.

Рассмотрим динамическую систему, математическая модель которой описывается стохастическим дифференциальным уравнением:

$$
\frac{d x}{d t}=F(t, x)+B(t) \xi(t),
$$

где $x-n$-мерный вектор состояния; $F(t, x)-n$ мерная гладкая вектор-функция; $B(t)$ - матрица порядка $n \times m$, элементы которой являются непрерывными функциями; $\xi(t)-m$-мерный белый шум с нулевым математическим ожиданием и заданной матрицей интенсивности $Q(t)$ порядка $m \times m$.

Начальные условия для системы (1) задаются априорным вектором $\bar{x}_{0}$ и его ковариационной матрицей $P_{0}$.

В моменты времени $t_{1}, t_{2}, \ldots, t_{N}$ производятся измерения функций $\Psi_{1}, \Psi_{2}, \ldots, \Psi_{N}$. Измеренное значение функции $\Psi_{i}$ обозначим через $\left(\Psi_{i}\right)_{\text {наб }}$. Для каждого момента времени $t_{i}$ справедливо

$$
\left(\Psi_{i}\right)_{\text {наб }}=\Psi_{i}\left(t_{i}, x(\cdot)\right)+\eta_{i},
$$

где $\eta_{i}-$ случайный вектор, имеющий нулевое математическое ожидание и ковариационную матрицу $R_{i}$.

Запись в качестве параметра $x(\cdot)$ функции $\Psi_{i}$ означает, что функция $\Psi_{i}$ зависит не от мгновенного значения вектора состояния, а от функции $x(t)$, которая является решением уравнения (1).

В линейной постановке уравнение (1) примет вид:

$$
\frac{d}{d t} x=A(t) x+B(t) \xi(t),
$$

где $A(t)$ - квадратная матрица порядка $n \times n$, элементы которой являются непрерывными функциями времени $t$.

Измеряемые функции $\Psi_{1}, \Psi_{2}, \ldots, \Psi_{N} \quad$ в линейной постановке являются линейными функциями вектора состояния $x(t)$. В каждый момент времени $t_{i}$ справедливо соотношение:

$$
z_{i}=H_{i}\left(t_{i}\right) \cdot x\left(t_{i}\right)+\eta_{i}
$$

где $z_{i}-$ вектор параметров размерности $r_{i}$, измеряемых в момент времени $t_{i} ; H_{i}\left(t_{i}\right)$ - матрица размерности $r_{i} \times n ; \eta_{i}-$ последовательность независимых случайных векторов, имеющих нулевое математическое ожидание и ковариационную матрицу $R_{i}$.

Зависимость между векторами состояния системы в дискретные моменты времени $t_{i}$, определяемая дифференциальным уравнением (3), может быть выражена при помощи его разностного аналога, определяемого соотношениями:

$$
x\left(t_{i+1}\right)=\Phi\left(t_{i+1}, t_{i}\right) x\left(t_{i}\right)+v\left(t_{i}\right) .
$$

Здесь $\Phi\left(t_{i+1}, t_{i}\right)$ - фундаментальная матрица, удовлетворяющая матричному уравнению

$$
\frac{d}{d t} \Phi\left(t, t_{i}\right)=A(t) \Phi\left(t, t_{i}\right),
$$

при начальном условии $\Phi\left(t_{i}, t_{i}\right)=E$, где $E-$ единичная матрица размерности $n$.

$$
\left\{v\left(t_{i}\right), i=0,1,2, \ldots, N\right\} \text { - последовательность }
$$

случайных векторов с нулевым математическим ожиданием и ковариационными матрицами $Q_{i}$, вычисляемыми по формуле:

$$
\begin{gathered}
Q_{i}=\int_{t_{i}}^{t_{i+1}} \Phi\left(t_{i+1}, \tau\right) B(\tau) Q(\tau) B^{T}(\tau) \Phi^{T}\left(t_{i+1}, \tau\right) d \tau .(7) \\
\text { Случайный } \quad \text { вектор } \quad v\left(t_{i}\right), i=0,1,2, \ldots, N
\end{gathered}
$$

связан с шумом $\xi(t)$ соотношением:

$$
v\left(t_{i}\right)=\int_{t_{i}}^{t_{i+1}} \Phi\left(t_{i+1}, \tau\right) B(\tau) \xi(\tau) d \tau .
$$

Он может быть интерпретирован, как суммарное воздействие возмущений на интервале времени от $t_{i}$ до $t_{i+1}$, т. е. между $i$-ым и $i+1-$ ым измерениями.

Введем следующие обозначения:

$$
\begin{aligned}
& x_{i}=x\left(t_{i}\right), \Phi_{i}=\Phi\left(t_{i+1}, t_{i}\right), \\
& Q_{i}=Q\left(t_{i+1}, t_{i}\right), v_{i}=v\left(t_{i}\right) .
\end{aligned}
$$

C учетом соотношения (4) и полученной системы разностных уравнений задача оценивания может далее рассматриваться в дискретной постановке. Требуется получить оценку вектора состояния $x_{i}$ дискретной динамической системы, которая описывается следующим соотношением:

$$
x_{i+1}=\Phi_{i} x_{i}+v_{i}, \quad i=0, \ldots, N, \ldots
$$

при априорно заданной оценке начального вектора состояния $\bar{x}_{0}$ и ковариационной матрице этой оценки $P_{0}$. Измеряемые величины $z_{i}, i=1, \ldots, N, \ldots$ связаны с векторами состояния уравнениями:

$$
z_{i}=H_{i} x_{i}+\eta_{i}, \quad i=1, \ldots, N, \ldots
$$


где $\eta_{i}$ - случайный вектор размерности $r_{i}$ с нулевым математическим ожиданием и ковариационной матрицей $R_{i}$.

Построим оценку вектора состояния и суммарных возмущающих воздействий дискретной динамической системы $(10,11)$ по мерной базе, содержащей $N$ измерений. Используем метод наименьших квадратов. Критерием качества оценки является квадратичная форма следующего вида:

$J=\frac{1}{2}\left(\hat{x}_{0, N}-\bar{x}_{0}\right)^{T} P_{0}^{-1}\left(\hat{x}_{0, N}-\bar{x}_{0}\right)+$

$+\frac{1}{2} \sum_{i=0}^{N-1}\left(z_{i+1}-H_{i+1} \hat{x}_{i+1, N}\right)^{T} R_{i}^{-1}\left(z_{i+1}-H_{i+1} \hat{x}_{i+1, N}\right)+\frac{1}{2} \sum_{i=0}^{N} \hat{v}_{i, N}^{T} Q_{i}^{-1} \hat{v}_{i, N}$,

где $\hat{x}_{i, N}$ - оценка вектора состояния на момент $t_{i}, i=0, \ldots, N$ с использованием $N$ измерений: $z_{1}, z_{2}, \ldots, z_{N} ; \hat{v}_{i, N}-$ оценка вектора суммарных возмущений $v_{i}$ при использовании $N$ измерений: $z_{1}, z_{2}, \ldots, z_{N}$.

Квадратичная форма содержит члены трех типов:

- квадраты невязок измеренных и расчетных значений, отнесенные к априорно известным среднеквадратическим отклонениям ошибок измерений;

- квадраты суммарных возмущений на интервалах между измерениями, отнесенные к априорно известным средним значениям суммарных возмущений на тех же интервалах;

- квадрат взвешенного отклонения априорно заданного вектора состояния от его расчетного значения.

Оценки $\hat{x}_{N, N}$ и $\hat{x}_{N-1, N-1}$, т. е. оценки векторов состояния на момент последнего измерения, полученные по $N$ и $N-1$ измерениям, связаны следующим рекуррентным соотношением:

$\hat{x}_{N, N}=\Phi_{N-1} \hat{x}_{N-1, N-1}+P_{N, N} H_{N}^{T} R_{N}^{-1}\left[z_{N}-H_{N} \Phi_{N} \hat{x}_{N-1, N-1}\right]$,

где матрица $P_{N, N}$ вычисляется по рекуррентным формулам:

$$
\begin{gathered}
P_{1,0}=\Phi_{0} P_{0} \Phi_{0}^{T}+Q_{0}, \\
P_{1,1}=\left(P_{1,0}^{-1}+H_{1}^{T} R_{1}^{-1} H_{1}\right)^{-1}, \\
P_{2,1}=\Phi_{1} P_{1,1} \Phi_{1}^{T}+Q_{1}, \\
P_{2,2}=\left(P_{2,1}^{-1}+H_{2}^{T} R_{2}^{-1} H_{2}\right)^{-1}, \\
\ldots \ldots \ldots \ldots \ldots \ldots \ldots \ldots \ldots \ldots \ldots \ldots \ldots \ldots \ldots \\
P_{N, N-1}=\Phi_{N-1} P_{N-1, N-1} \Phi_{N-1}^{T}+Q_{N-1}, \\
P_{N, N}=\left(P_{N, N-1}^{-1}+H_{N}^{T} R_{N}^{-1} H_{N}\right)^{-1} .
\end{gathered}
$$

Оценки вектора состояния $\hat{x}_{i, N}$ и суммарного воздействия возмущений $\hat{v}_{i, N}$ на момент $t_{i}$ вычисляются с использованием следующих рекуррентных соотношений:

$$
\begin{gathered}
\lambda_{N-1}=H_{N}^{T} R_{N}^{-1}\left[z_{N}-H_{N} \hat{x}_{N, N}\right] ; \\
\hat{v}_{N-1, N}=Q_{N-1} \lambda_{N-1} ; \\
\hat{x}_{N-1, N}=\Phi_{N}^{-1}\left[\hat{x}_{N, N}-\hat{v}_{N-1, N}\right] ; \\
\lambda_{i}=\Phi_{i+1}^{T} \lambda_{i+1}+H_{i+1}^{T} R_{i+1}^{-1}\left[z_{i+1}-H_{i+1} \hat{x}_{i+1, N}\right] ; \quad(16) \\
\hat{v}_{i, N}=Q_{i} \lambda_{i} ; \\
\hat{x}_{i, N}=\Phi_{i}^{-1}\left[\hat{x}_{i+1, n}-v_{i, N}\right], \quad \text { для } i=N-2, \ldots, 0 . \\
\text { Здесь } \quad \lambda_{N-1,} \lambda_{N-2, \ldots,} \lambda_{0} \quad-\text { вспомогательные }
\end{gathered}
$$

векторы.

\section{5. Выводы}

В этой работе были проанализированы основне типы и виды отказов. Было выделено три основные группы отказов, таких как отказы датчиков, отказы приводов и отказы компонентов систем. Так же был разработан метод диагностирования возникновения отказа. Используя данный метод в системе диагностирования отказов, можно будет установить тип и место отказа. Что существенно увеличит отказоустойчивость самолета, а так же его устойчивость и управляемость. Как следствие позволит осуществлять более удачную реконфигурацию управления самолетом.

\section{Литература}

1. Биргер, И. А. Техническая диагностика [Текст] / И. А. Биргер. - М. : Машиностроение, 1978. - 240 с.

2. Дмитренко, И. Е. Техническая диагностика и автоконтроль [Текст] / И. Е. Дмитренко. - М. : Транспорт, 1986. $-144 \mathrm{c}$.

3. Сфарбаков, А. М. Основы технической диагностики [Текст]: уч. пос. / А. М. Сфарбаков, А. В. Лукьянов, С. В. Пахомов. - Иркутск : ИрГУПС, 2006. $-216 \mathrm{c}$.

4. Васильев, В. И. Многоуровневое управление динамическими объектами [Текст] : монография / В. И. Васильев. - М.:Наука, 1987. - 309 с.

5. Машков, О. А. Обнаружение отказов в системе управления на основе различения многих гипотез [Текст] / О. А. Машков, О. Е. Гиро, П. Б. Щавелев // Оборудование летательных аппаратов. - 1993. - Ч. III. - С. 32-35.

6. Скрипниченко, В. Г. Применение математического моделирования и теоретических методов при анализе особых случаев взлета и посадки воздушных судов [Текст] : дисс. ... докт. техн. наук / В. Г. Скрипниченко. - М, 2005. -438 с.

\section{References}

1. Birger, I. A. (1978). Technicheskaya diagnostika [Technical diagnostics]. Moscow: Mashinostroenie, 240.

2. Dmitrenko, I. E. (1986). Tehnicheskaya diagnostika I avtokontroly [Technical diagnostics and automonitoring]. Moscow: Transport, 144.

3. Sfarbakov, A. M., Luk'yanov, A. V., Pahomov, S. V. (2006). Osnovi technicheskoy diagnostiki [Base of Technical diagnostics]. Irkutsk: IrGUPS, 216.

4. Vasilyev, V. I. (1978). Mnogourovnevoye upravlenie dinamicheskimi ob'ektami [Dinamical objects multilevel control]. Moskow: Scince, 309. 
5. Mashkov, O. A., Giro, O. E., Shavelev, P. B. (1993). Obnarujenie otkazov $\mathrm{v}$ sisteme upravleniya na osnove razlichiya mnogih gipotez [Fault detection in control system using many hypotheses diference]. Oborudovanie letalynih aparatov [Aircraft equipment], 3, 32-35.
6. Skripchenko, V. G. (2005). Primeneniye matematicheskogo modelirovaniya I teoretycheskych metodov pri analyze osobich sluchayev vzleta I posadki vozdushnih sudov [Mathematical modeling and theoretical methods usage for extra situation analyze on aircraft take off and landing stage]. Moscow, 483.

Дата надходження рукопису 30.11.2014

Казак Василий Николаевич, доктор технических наук, профессор, кафедра автоматизации и энергоменеджмента, Национальный авиационный университет, пр. Комарова, 1, г. Киев, Украина, 03058 E-mail: profkazak@ukr.net

Шевчук Дмитрий Олегович, кандидат технических наук, докторант, кафедра автоматизации и энергоменеджмента, Национальный авиационный университет, пр. Комарова, 1, г. Киев, Украина, 03058 E-mail: dmitroshevchuk@gmail.com

Васильев Михаил Андреевич, аспирант, кафедра автоматизации и энергоменеджмента, Национальный авиационный университет, пр. Комарова, 1, г. Киев, Украина, 03058

E-mail: vasilyev_ma@ukr.net

\section{УДК 004.89}

DOI: 10.15587/2313-8416.2014.32910

\section{ПРИНЦИПЫ ПОСТРОЕНИЯ ИНФОРМАЦИОННОЙ ТЕХНОЛОГИИ СБОРА И СИСТЕМАТИЗАЦИИ БИЗНЕС-ИНФОРМАЦИИ}

\section{(C) Ю. Н. Гонтарь}

Особенность бизнес-информации, которая используется на предприятии, заключается в ее больших объемах и неструктурированном виде. В силу этого проиесс принятия решений на основе этой информации зачастую происходит не эффективно. Важнейтими задачами в этом случае являются систематизация бизнес-информации, анализ и повторное использование собранных данных. В данной статье были выделены основные принципы построения информационных технологий для решения этих задач

Ключевые слова: бизнес-среда, бизнес-процесс, бизнес-информация, информационнье технологии, систематизащия, классификаџия, каталогизация, репозиторий, онтология

The special feature of business-information at the enterprise is about its high-volume and unstructured formats. Therefore decision-making process on the basis of this information often does not occur effectively. The most important tasks in this case are systematization of business-information, analysis and re-use of collected data. This article presents the basic principles of information technology to solve these problems

Keywords: business-environment, business-processes, business-information, information technology, systematization, classification, cataloging, repository, ontology

\section{1. Введение}

Современные модели сложных систем обработки информации, технологии и инструментальные средства создания программных продуктов, описания их функционирования столь разнообразные и мощные, что возникает иллюзия простоты их применения. Однако важнейшим моментом реализации таких систем является определение принципов построение модели системы, которая бы наиболее адекватно описывала тонкости eе работы и предоставляла удобные средства и механизмы исследования желаемых свойств.

За последние годы произошло значительное распространение различных Интернет-сервисов. Но технология совместной работы, основанной на специальном программном обеспечении и среде взаимодействия для совместного решения конкретных задач (так называемое collaboration environment), развивается достаточно медленно [1]. В обычном понимании электронный бизнес выглядит как поток транзакций, циркулирующих внутри организаций и между ними для поддержки бизнес-процессов. Несмотря на значительную привлекательность существующих технологий обработки электронных транзакций, организации подстерегает ряд проблем, решить которые способны только новые методы. К ним можно отнести такие как несовместимость форматов данных, консолидация бизнеса, управления распределенными корпоративными данными [2].

Современный Интернет не является интеллектуальным по своей природе, так как хранит не чистые знания, а данные, из которых знания нужно получать эвристическими методами. Это приводит к ряду актуальных научных задач, одной из которых является систематизация бизнес-инфор-мации. Под термином «бизнес-информация» обычно понимается существенная информации, которая непосредственно связана с деятельностью предприятия и его бизнес- 